NASA Technical Memorandum 88953

\title{
Laser Anemometry Techniques for Turbine Applications
}

Mark P. Wernet and Lawrence G. Oberle

Lewis Research Center

Cleveland, Ohio

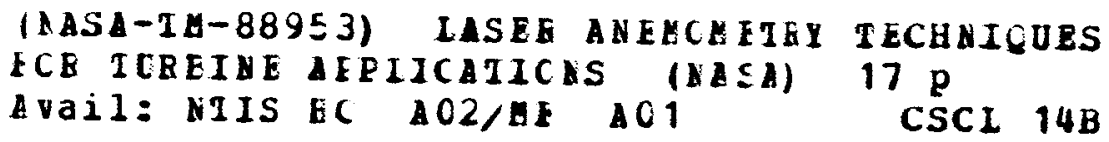

187-22959

$\begin{array}{ll}\text { 65 } & \text { Unclas } \\ 4 / 35 & 0076795\end{array}$

Prepared for the

32nd International Gas Turbine Conference and Exhibition sponsored by the American Society of Mechanical Engineers Anaheim, California, May 31-June 4, 1987
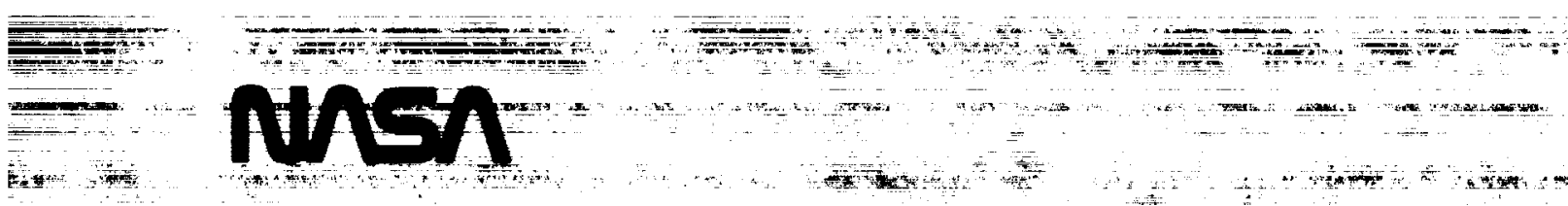


\section{LASER ANEMOMETRY TECHNIQUES FOR TURBINE APPLICATIONS}

Mark P. Wernet and Lawrence G. Oberle

Nationa 1 Aeronautics and Space Administration

Lewis Research Center

Cleveland, Ohio 44135

\section{ABSTRACT}

Laser anemometry offers a nonintrusive means for obtaining flow field information. Our current research efforts at NASA Lewis Research Center are focused on instrumenting a warm turbine facility with a laser anemometer system. In an effort to determine the laser anemometer system best qualified for the warm turbine environment, we compared the performance of a conventional laser fringe anemometer and a two spot time-offlight system with a new, modified time-of-flight system, called a Four Spot laser anemometer. The comparison measurements were made in highly turbulent flows near walls. The four spot anemometer uses elliptical spots to increase the flow acceptance angle to be comparable to that of a Laser Fringe Anemometer. Also, the four Spot uses an optical code that vastly simplifies the pulse detection processor. The results of the comparison measurements will exemplify which laser anemometer system is best suited to the hostile environment typically encountered in warm rotating turbomachinery.

\section{INTRODUCTION}

A laser anemometer offers a nonintrusive method for obtaining flow fleld information. Particles entrained in the flow provide scattering centers for the incident light. There are two common techniques for opticaliy coding the measurement region. The Laser Fringe Anemometer (LFA) employs a sinusoldally varying fringe pattern. Knowledge of the fringe spacing and the detected signal frequency of particles traversing the measurement region permits the determination of the velocity component normal to the fringes. Another technique for encoding the measurement region (Time-ofFlight Anemometer, TOFA) uses two closely spaced spots, where the flow velocity component parallel to the axis of the spots is obtained from the time-of-flight of particles traversing the two spots.

The motivation for this work was the desire for an anemometer capable of measurements near walls in turbulent flows. This requires a laser anemometer with special qualities. The optimum anemometer would have a wide acceptance angle to enable measurements of wide flow angle variations, and also have a high spatial selectivity, to limit unwanted flare light scattered from surfaces reaching the detector. The LFA typlcally has a wider acceptance angle than a conventional TOFA system. This reduces the utility of TOFA systems in turbulent flows. However, the TOFA receiver can have much better spatial resolution than the LFA for the same $f$ /number system.

Our current research efforts at NASA Lewis are focused on instrumenting a $50.8 \mathrm{~cm}$ diameter, single stage, warm turbine facility with a laser anemometer system. In an effort to determine the laser anemometer system best qualified for the warm turbine environment, we compared the performance of conventional LFA and TOFA systems with a new, modified TOFA system, called a Four Spot laser anemometer. The comparison measurements were made in highly turbulent flows near walls. The Four Spot TOFA uses elliptical spots to increase the flow acceptance angle to be comparable to that of an LFA. Also, the Four spot uses an optical code that vastly simplifies the pulse detection processor. The results of the comparison will exemplify which laser anemometer system is best suited to the hostile environment typically encountered in warm rotating turbomachinery.

\section{FOUR SPOT LASER ANEMOMETER}

The electronic signal obtained from a normal two spot TOFA system consists of two notsy gaussian shaped pulses separated by the transit time of a particle traversing the two light spots in the measurement region. An estimate of the peak to peak time-of-flight and knowledge of the spot spacing then yields the particle velocity component along the axis of the two spots. The inherent noise on any type of photon detection system decreases the ability of the signa] processor to determine the exact time-of-occurrence of a pulse. The particle's time-of-flight can be obtained more accurately by transforming the unipolar pulse into a bipolar pulse. The zero crossings of the bipolar pulses yield the estimated time of flight. The transformation from a unipolar to a bipolar pulse should not introduce additional noise to the signal. Lading (1) analyzed the performance of four methods for generating 
bipolar pulses: the derivative operator, Hilbert transforms, and both the spatial and temporal lead-lag filter. The results of his analysis showed that an advantage could be obtained by implementing the leadlag filter spatially, before photon detection. The advantage of the spatial lead-lag filter is that the dimensions are fixed in space, but the temporal scale of the signal depends on the velocity. Thus, a spatial implementation $w \nmid 11$ behave as an adaptive temporal lead-lag operator. The transformation to a bipolar pulse is thus made without adding noise to the signal and in a robust manner that does not depend on electronic delays. The Four Spot Anemometer system described herein has a spatial lead-lag filter (2).

The Four Spot TOFA system uses two pairs of partially overlapping spots in the measurement region. These two pairs of spots, labeled $A$ to $D$, are separated by a distance $X_{0}$, orthogonally polarized, and partially overlapping (Fig. 1). The amount of overlap is, $2 \sigma$, where $\sigma$ is the standard deviation of $e^{-2}$ gaussian spot width. The use of elliptical spots increases the acceptance angle of the measurement region - comparable to that of a laser fringe anemometer.

The transmitting section of the system contains two quarter-wave plate/Wollaston prism pairs (Fig. 2) The input light is linearly polarized. A cylindrical lens $L_{1}$ transforms the circular input beam into an elliptical beam. The first quarter-wave platel Wollaston prism pair $Q_{1} / W_{1}$ generates two angularly diverging, orthogonally polarized beams. These plane polarized beams are imaged by $L_{2}$ and $L_{3}$ onto the second pair $Q_{2} / W_{2}$. The first pair $Q_{1} / W_{1}$ must be at the back focal plane of $L_{2}$, and $Q_{2} / W_{2}$ must be at the front focal plane of $L_{3}$ to maintain the sharpness of the imaged spots. Emerging from the $\mathrm{Q}_{2} / \mathrm{W}_{2}$ pair are four consecutively, orthogonally polarized beams. The angular divergence of these beams is transformed into a spatial separation by the lens $L_{4}$. The angular divergence imparted by the $0_{1} / W_{1}$ pair creates the spatial separation $x_{0}$. The angular divergence imparted by $\mathrm{O}_{2} / \mathrm{W}_{2}$ creates the partially overlapping spots in the measurement region.

The measurement region geometry is controlled by the input beam diameter, the angular divergences of $W$ and $W_{2}$, and by the focal length of $L_{4}$. The quarterwave plates allow the equalization of the intensities of the four spots.

The backscatter system configuration collects the scattered light from the measurement region back along the axis of the transmitted beam. The use of two elevation mirrors $M_{1}$ and $M_{2}$ allows this coaxial configuration. The received image is magnified by the image pair $L_{4}$ and $L_{7}$. The rectanguiar mirror $M_{2}$ acts as a vertical spatial filter mask in the receiver. The received light is recombined into two pairs of totaliy overlapping spots by a third wollaston prism, $W_{3}$, which has the same angular split as $W_{2}$. These two spots are then imaged onto the receiver mask consisting of two precision air slits. The two totally overlapping pairs of spots are separated by a polarization selective beam-splitting cube. Two right angle mirrored prisms are used to separate the spot pairs into four individual signals. The separated signals are detected by four RCA 8645 photomultiplier tubes.

The Four Spot TOFA has been constructed using optical erector components (Fig. 3). A mirror-type image rotator has been incorporated into the Four Spot TOFA. The image rotator is common to both the transmitter and receiver, and permits two-dimensional velocity scans by taking measurements at several angular orientations of the measurement volume.
The temporal separation of the bipolar pulses is determined by a high speed Emitter-Coupled Logic (ECL) zero crossing detection circuit. The ECL processor outputs start and stop puises which are fed into a time analyzer. The start and stop pulses correspond to a particle traversing the two pairs of spots in the measurement region, that is, the time-of-flight. The time analyzer generates a voltage pulse ( 0 to $10 \mathrm{~V}$ ) that is proportional in amplitude to the time difference between the start-stop pulses. These voltage pulses are then sent to an analog to digital converter $(A D C)$. The digital words from the $A D C$ are fed into the back plane of a TSI model 1998 master interface. The TSI module generates a time-between-data (TBD) word from the Four spot data. Both the measured velocity and TBD are sent via a Direct Memory Access interface to a PDP $11 / 44$ computer for analysis. At each measurement position, 1000 velocity events and 1000 TBDs are recorded.

\section{LASER FRINGE ANEMOMETER}

The LFA system used in these comparison measurements was set up to enable good flare light rejection performance in a coaxial backscatter arrangement. The specific operating parameters of the system are listed in Table 1. The LFA system also made use of a signal preprocessor, which was designed and constructed at Lewis Research Center. The signal preprocessor replaces the input conditioning tasks of the countertype processor. The preprocessor allows selection of the high voltage for the photomultiplier tube, high pass and low pass filters, signal attenuation, and monitoring of the photomultiplier tube dc current level, all via the system computer. The menu driven preprocessor software is incorporated into the laser anemometer system velocity survey software. The laser anemometry software also allows on-line histogram displays for validation of the data and the system parameters. The values of the system parameters selectable through the preprocessor are archived along with the measured histoarams and time-between-data information.

\section{TWO SPOT ANEMOMETER}

A conventional Two Spot TOFA system using a correlation scheme (3) for signal processing was avallable at NASA Lewis, but was not operational at the time of this work. A Two spot anemometer type system was obtained by removing the cylindrical lens from the four Spot TOFA. This reduced the acceptance angle of the Four Spot to be on the order of a conventional Two Spot system. The electronics and signal processing scheme were the same as those used in the Four Spot system. Throughout the remainder of the text, this rOFA system with the small acceptance angle will be referred to as a Two Spot TOFA. This is a better comparison than would have otherwise been available. All three systems were using the same collection optics. Both TOFAs were using identical signal processing schemes, the only difference between these two TOFA systems was the probe volume acceptance angle.

Table 1 lists the important operating characteristics of each of the laser anemometer systems in the comparison. A Bragg cell was added to the LFA system to enable the measurements of the reversing flows in the blade wake. All three systems were capable of bidirectional velocity measurements. The probe volume dimensions were measured using a pinhole/photomultiplier tube assembly. The LFA probe volume dimensions were determined by measuring the beam diameter and separation before the final focusing lens. The fringe spacing and probe volume diameter were then calculated 
from these measured values. The TOFA probe volumes were scanned over the pinhole and the recorded photomultiplier tube dc current as a function of position was fit to a gaussian using the nonlinear least squares technique. For the TOFA systems, the e-2 widths and heights of the fitted gaussians were taken as the probe volume dimensions. The spot pair separations of the TOFAs were determined from the difference in the peak locations of the measured spots. The average light flux through the probe volumes were calculated using the probe volume on-axis cross-section dimensions and the laser power given in Table 1 . For the LFA, a circular probe volume cross-section was assumed, with only 50 percent lllumination, in order to account for the bright and dark fringes. Four circular spots were used to calculate the average light flux in the Two Spot TOFA. The Four Spot average light flux value was calculated assuming four 117 uminated ellipses, with major and minor axes given as probe volume height and spot width respectively.

Each of the laser anemometer systems was setup on an optical breadboard mounted on a three-axis positioning system. The laser anemometry software controls the positioning system. The three-axis table enabled velocity surveys along a neariy normal line to the suction surface of the rotor blade. All three laser anemometer systems were 1-component systems with beam rotation or image rotation capabilities for obtaining two-dimensional surveys in the high turbulence regions.

The LFA and the Four spot systems were constructed using the same $f / 2.5$, diffraction 1 imited, $100 \mathrm{~mm}$ diameter, collection optics. The use of low f/number collection optics permitted the use of large aperture masks in the receiving optics. Larger spatial filtering masks increase the system's spatial selectivity. The LFA and TOFA systems were implemented with mask to lens clear aperture ratios of 50 percent. All three laser anemometer systems used an argon ion laser operating at the $514.5 \mathrm{~nm}$ wavelength.

\section{EXPERIMENTAL SETUP AND CALIBRATION}

The comparison tests were performed in an open jet burner facility. The burner rig exit nozzle was approximately $7.5 \mathrm{~cm}$ in diameter. The burner $\mathrm{rig}$ was seeded with refractory alumina particles supplied via a commercial fluidized bed seed generator. The mean seed particle diameter, measured using a commercial aerosol particle sizer, was approximately $1 \mu \mathrm{m}$. Typical mean velocities, temperatures, and turbulence intensities obtainable with this burner rig were approximately $300 \mathrm{~m} / \mathrm{sec}, 700^{\circ} \mathrm{C}$, and 6 percent, respectively.

In order to generate higher turbulence and supply a surface for the surface proximity measurements, a single turbine rotor blade was mounted in the burner flow, as shown in Fig. 4. The blade used in these comparison measurements was a solid, core turbine rotor blade, with an axial cord of $3.429 \mathrm{~cm}$, a leading edge radius of $0.298 \mathrm{~cm}$, a tralling edge radius of $0.089 \mathrm{~cm}$, and a height of $3.81 \mathrm{~cm}$. The blade was mounted $3 \mathrm{~cm}$ downstream, centered in the nozzle exit plane, and oriented to within $2^{\circ}$ of the design inlet flow angle. The comparison measurements were taken on the suction side of the rotor blade. See Fig. 4 for locations of the measurement stations and the reference coordinate system. This geometry provided a good'source of highly turbulent flow. The region near the blade leading edge would provide the environment for the one-dimensional surface proximity measurements, since the blade surface was almost normal to the laser anemometer optical axis in this region. The region near the trailing edge of the blade would provide a highly turbulent region, requiring two-dimensional surveys, for determining the turbulence performance capabilities of these laser anemometer systems.

These comparison measurements should show that both of the TOFA systems perform better near surfaces than the LFA, due to their superior flare light rejection capabilities. In the more turbulent flow, near the blade trafling edge, the systems with large acceptance angle should show better performance (LFA and Four Spot) than the small acceptance angle system. Ideally, these experiments should show that the Four Spot TOFA has the required properties to enable measurements under both types of conditions.

In order to verify the surface proximity measurements, the blade surface location had to be determined for each survey position. The surface location was determined by measuring the dc current from the photomultiplier tube in the laser anemometer receiver as the probe volume was scanned through the blade surface. These scan profiles were then fit to a gaussian function by the method of nonlinear least squares. The gaussian peak location was assumed to be the actual blade surface location. For both TOFAs, the scans were performed twice, once on each spot pair, and the average peak position from the two scans was taken as the surface location. The maximum difference in the surface location determined using both spot pairs was $80 \mu \mathrm{m}$. The origin of the reference coordinate system was placed at the top leading edge of the blade.

The angular orientations of the probe volumes were determined by projecting the probe volumes onto a screen via a microscope objective. A verticle line on the screen served as a reference for determining the $0^{\circ}$ probe volume angular orientation. (A better technique would have been to measure the spot, or fringe position as a function of probe volume height. This would yield the probe volume angular offset.) Any errors in the angular calibration between the LFA and the TOFA would yield a constant angular offset in the measured flow angle data. The offset would be constant, and unimportant. The general shape of the flow angle measurements would be illustrative of each laser anemometer systems flow measurement capabilities. The Four Spot and the Two Spot systems used the same refertnce angle, since the only difference between these two systems was the removal of the cylindrical lens. The flow angle data obtained from these two systems should be identical, but there may be a constant offset between the LFA and both TOFA system's flow angle data sets.

\section{RESULTS AND DISCUSSION}

There were two types of comparison measurements made in the burner rig. Those near the leading edge, which will be called surface proximity measurements, and those towards the trailing edge of the blade, which will be referred to as flow angle measurements. The surface proximity measurements are measures of how close to a surface a laser anemometer system can obtain velocity measurements. Since both TOFA systems would have performed the same for the surface proximity measurements, only data from the Four Spot TOFA and the LFA will be discussed. All three systems were used in the flow angle measurements, since the four spot and Two Spot systems do not have the same acceptance angles. All measurements were made in the $Y=-17.5 \mathrm{~mm}$ plane, which is approximately mid height on the blade.

\section{Surface Proximity Measurements}

The surface location scans were used to determine the surface locations prior to the surface proximity 
surveys. The surface proximity surveys were performed at stations 2 through 8 along the Z-axis, see Fig. 4 , in the $X$-axis direction. The surface location surveys also yielded an indication of the effective on-axis probe volume length via the $e-2$ width of the gaussian intensity curves. The scans indicated that the LFA and TOFA systems had effective on-axis probe volume lengths of 1.16 and $0.22 \mathrm{~mm}$ respectively. These on-axis probe volume lengths can be viewed as an indicator of a particular laser anemometer system's ability to obtain measurements near surfaces.

The results of the surface proximity measurements are shown in Fig. 5. The LFA and Four Spot TOFA minimum approach distances along the $X$-survey direction are shown along with the actual blade surface. The minimum approach distance is the distance from the blade surface at which the last velocity measurement could be obtained. Notice that the surveys at stations 2 through 5 were not at normal incidence, but those surveys at stations 6 through 8 were at near normal incidence. The graph shows qualitatively the performance of each system over the range of the blade surface. At all but one station, no. 6 , the Four Spot was able to obtain measurements at least twice as close to the surface than the LFA system. One factor affecting these measurements is the surface reflectivity. The blade surface was a plain brushed metal finish; no special coatings or paints were used. Seed particle bulldup on the surface of the blade may have altered the surface reflectivity in some regions. Both data sets were obtained with an initially clean blade surface. Another factor affecting the surface proximity measurements is the lack of sufficient seed particles at the surface. The low seed particle concentration near the surface is an ultimate limit on these surface proximity measurements. Considering the same conditions for both laser anemometer systems, the results show that the four Spot TOFA has superior flare light rejection capabilities compared to the LFA. For surface proximity measurements, the TOFA would yield better performance than an LFA.

An interesting flow phenomenon was observed in the surface proximity measurements at station no. 3, $2 \mathrm{~mm}$ from the blade leading edge. The LFA measurements at this survey station yielded no unexpected results single peaked histograms to within $700 \mu \mathrm{m}$ from the blade surface. However, the Four Spot TOFA histograms showed an oscillating flow phenomenon beginning at about $1300 \mu \mathrm{m}$ from the surface and continuing in to $330 \mu \mathrm{m}$ from the surface. Figures $6(\mathrm{a})$ through $6(\mathrm{e})$ show the double peaked histograms, indicating a flow oscillation. The blade surface is at $3.27 \mathrm{~mm}$ at this survey station, the $X$-positions in the figures can be referenced to this surface location. The large lobe, which represents the steady state flow, remains relatively constant as the side lobe grows in size, and then decreases again close to the surface. Oiscussions with several fluid dynamicists revealed that this bifurcating flow is not uncommon near the blade leading edge. This phenomenon appeared to be unresolvable with the LFA due to its poor flare light rejection and its longer on-axis probe volume length. The longer probe volume length causes the LFA to average a larger cross section of the flow, which may have caused the steady state flow signal to swamp the bifurcating flow signal. Hence, we see that the high spatial selectivity in TOFA systems is preferabie in a case such as this for more clearly resolving flow features.

\section{Flow Angle Measurements}

The flow angle measurements were made at station no. 9 in Fig. $4,20 \mathrm{~mm}$ downstream from the blade leading edge. At this distance from the blade leading edge, the blade surface has turned in sharply and a recirculation zone would be expected. The Bragg shifted LFA system was used to obtain a standard reference data set at survey station no. 9. At each measurement position, the mean velocity was measured at three angular orfentations of the probe volume, $\pm 15^{\circ}$, and $0^{\circ}$. The LFA data was assumed to have a cosine dependence on the relative angle between the mean flow direction and the probe volume angular ortentation (4). The velocity magnitude and flow angle were estimated from the mean velocity data as a function of probe volume angle by the method of nonlinear least squares. The LFA flow angle data was used as a reference data set for the Two Spot and Four Spot TOFA data.

The same technique for reducing the LFA flow angle data was used on the Four Spot TOFA data. Again, three angular orientations, $\pm 75^{\circ}$, and $0^{\circ}$, of the probe volume were used. The assumption of the cosine dependence for this TOFA system was used since the Four Spot has a large acceptance angle, similar to that of an LFA.

The low acceptance angle of the Two Spot system required that a different data reduction scheme be used. The Two Spot system does not have the same sampling statistics as an LFA system. The flow angle data taken with the Two Spot required more angular orientations of the probe volume in order to resolve the flow angle, due to the high turbulence flow. Seven angular orientations of the probe volume were used with a $3^{\circ}$ interval (5). The mean velocities were calculated from the collected histograms at each measurement angle. The data taken with a small acceptance angle anemometer does not have a cosine dependence on the measurement angle. The small acceptance angle of this system results in a measured histogram, which is a thin slice out of the two-dimensional gaussian velocity probability distribution. So the collected data are one-dimensional histograms, or slices of a twodimensional gaussian at different angles. The mean values calculated from the measured histograms are points on a one-dimensional gaussian distribution, as a function of angle. Hence, the Two Spot flow angle data was reduced by fitting the measured mean velocities tc a gaussian function of angle (6).

The turbulence intensities were calculated for all three data sets. The turbulence intensity, as referred to in this text, includes the measurement uncertainty, from the measured histograms, and the inherent instrument error. Isotropic turbulence was assumed, so that the LFA and Four Spot TOFA turbulence intensities, at all three angular orientations of the probe volume, could be averaged to obtain an estimate of the turbuience intensity as a function of survey position. For the Two Spot TOFA flow angle data, only the data at the three angular orientations centered about the estimated flow angle were averaged to estimate the turbulence intensity.

In these comparison measurements, the operating parameters of the burner rig were identically set for each run, but some varlations in the mean flow magnitude, between runs, did occur. These run-to-run variations, which were on the order of a few percent, should not affect the trends observed in the collected data. Again, the burner $\mathrm{r}^{\mathrm{lg}}$ was used to provide a high turbulence flow, simflar to that expected in a warm turbine environment.

The comparisons of the flow angle data obtained with all three anemometer systems are shown in Figs. 7 to 9 . Figure 7 shows the comparison of the estimated velocity magnitudes. The difference in the absolute values of the velocity magnitude is the result of the burner rig mean velocity variation between runs. 
Although the operating parameters of the burner rig were set the same for all the runs, these mean velocity variations still occurred. The general velocity magnitude fluctuations in the three curves are very simllar up to $X=9 \mathrm{~mm}$. At $X=8 \mathrm{~mm}$, both the TOFA systems still show consistent variations in the velocity magnitude, but the LFA estimate shows a substantial drop in the velocity magnitude. At this survey position in the flow, a very sharp velocity gradient is present. This is the edge of the recirculation zone. The longer probe volume length in the LFA causes the estimate of the velocity magnitude at this measurement position to be blased to a lower value. Both TOFA system probe volumes, due to their shorter lengths, have not begun to detect much of this velocity gradient at this position. This assumption of the velocity gradient biasing is supported by the turbulence intensity data in Fig. 8. The comparison of the turbulence intensity for the three anemometer systems shows an average value of approximately 8 percent up to $X=9 \mathrm{~mm}$. At $X=8 \mathrm{~mm}$, the LFA turbulence intensity has substantially increased, while the TOFA systems only exhibit a silight rise in the turbulence intensity. The velocity gradient has broadened the LFA histograms, ratsing the estimated turbulence intensity. Due to their high spatial selectivity, the TOFA systems could yield a higher resolution picture of the flow, as is evidenced by these results.

Figure 9 shows a comparison of the estimated flow angle for the three anemometer systems as a function of survey position. Again, the LFA data is assumed to be a reference data set. The TOFA systems both used the same image rotator. The difference in the set up of these two systems was only in the use of a cylindrical lens for elliptical spot generation. The image rotator was not recalibrated between the change over from the Four Spot to the Two Spot TOFA. Any calibration errors in the probe volume angular orientation between the LFA and the TOFA systems should be systematic, but there should be no systematic errors between the Four Spot and Two Spot TOFAs' flow angle measurements.

The data in Fig. 9 shows a systematic calibration error in the probe volume angular orientation between the LFA and TOFA systems. The error in the calibration is approximately $2^{\circ}$ between the LFA and both TOFA systems. The shape of the Four Spot TOFA flow angle data agrees very closely with the LFA results throughout the survey range. However, the Two Spot flow angle data shows a random deviation from both the LFA and Four spot data. The fact that the velocity magnitude and turbulence intensity data for both of the TOFA systems agreed very closely supports the conclusion that this flow angle deviation is not a systematic error induced by the data reduction scheme.

As an alternative check, the Two Spot data rates were used to determine the flow angle, instead of the measured velocities (3). The Two Spot data rates were much more sharply peaked gaussian functions of the measurement angle than are the measured velocities. The sharper peaks should yield a better resolved flow angle estimate. The flow angle estimates using this technique are shown in Fig. 10. The magnitudes of the variations in the Two Spot flow angle data have been reduced, but the data does not exhibit the same behavior as the LFA and Four Spot flow angle estimates. These results indicate that questionable flow angle estimates are derived from small acceptance angle laser anemometer systems used in high turbulence environments.

\section{CONCLUSION}

The results of these comparison measurements show that for a high turbulence flow field, near surfaces, the Four Spot TOFA has shown superior performance over an LFA. This system appears best suited for the turbomachinery environment we will be investigating at Lewis Research Center. The TOFA systems, in general, show very good spatial selectivity in resolving flow features. Care shouid be taken when using small acceptance angle laser anemometer systems in high turbulence flows. These systems may not be capable of determining the true flow direction.

These results also indicate that, in a low turbulence flow, the elliptical spots in the four Spot measurement volume can be converted to circular spots, and the system would have a higher light flux through the measurement region. The higher light flux would make the system more sensitive to smaller particles.

\section{REFERENCES}

1. Lading, L., "Estimating Time and Time-Lag in Timeof-Filght Velocimetry," Applied Optics, Vol. 22, Nov. 15, 1983, pp. 3637-3643.

2. Wernet, M.P., and Edwards, R.V., "Implementation of a New Type of Time-of-Flight Laser Anemometer," Applied Optics, Vol. 25, No. 5, Mar. 1, 1986, pp. 644-648.

3. Smart, A.E., "Special Problems of Laser Anemometry in Difficult Applications," Laser Optical Measurement Methods for Aero-Engine Research and Deve lopment, AGARD-LS-90, AGARD, Par 15 , France, 1977, pp. 6-1 to 6-18.

4. Goldman, L.J., and Seasholtz, R.G., "Comparison of Laser Anemometer Measurements And Theory in an Annular Turbine Cascade With Experimental Accuracy Determined by Parameter Estimation" Engineering Applications of Laser Velocimetry, H.W. Coleman and P.A. Pfund, eds., ASME, NY, 1982, pp. 83-92.

5. Schod1, R., "A Laser-Two-Focus (L2F) Velocimeter For Automatic Flow Vector Measurements in the Rotating Components of Turbomachines," Measurement Methods in Rotating Components of Turbomachinery, B. Lakshminarayana and P.W. Runstadler, eds., ASME, NY, 1980, pp. 139-147.

6. Schodl, R., "A Laser Dual Beam Method For Flow Measurements in Turbomachines," ASME Paper 74-GT-157, Mar. 1974. 
TABLE 1.

\begin{tabular}{|c|c|c|c|}
\hline Parameter & LFA & Two-Spot & Four-Spot \\
\hline $\begin{array}{l}\text { Laser power } \\
\text { Collection optic diam } \\
\text { f/number } \\
\text { Probe volume width }(e-2) \\
\text { Probe volume helght }(e-2) \\
\text { Fringe size } \\
\text { Spot width } \\
\text { Average light flux } \\
\text { Acceptance angle } \\
\text { Signal processor }\end{array}$ & $\begin{array}{l}1.5 \mathrm{~W} \\
100 \mathrm{~mm} \\
2.5 \\
50 \mu \mathrm{m} \\
50 \mu \mathrm{m} \\
5.8 \mu \mathrm{m} \\
-- \\
1.5 \mathrm{E} \mathrm{W} / \mathrm{m}^{2} \\
> \pm 45^{\circ} \\
\text { Counter }\end{array}$ & $\begin{array}{c}1.5 \mathrm{~W} \\
100 \mathrm{~mm} \\
2.5 \\
106 \mu \mathrm{m} \\
10 \mu \mathrm{m} \\
10 \mu \mathrm{m} \\
4.8 \mathrm{Eg} \mathrm{W} / \mathrm{m}^{2} \\
\pm 6^{\circ} \\
\text { Custom design }\end{array}$ & $\begin{array}{c}1.5 \mathrm{~W} \\
100 \mathrm{~mm} \\
2.5 \\
106 \mu \mathrm{m} \\
97 \mu \mathrm{m} \\
10 \mu \mathrm{m} \\
0.5 \mathrm{Eg} \mathrm{W} / \mathrm{m}^{2} \\
\pm 43^{\circ} \\
\text { Custom design }\end{array}$ \\
\hline $\begin{array}{l}\text { Bidirectional } \\
\text { detection } \\
\text { scheme }\end{array}$ & $\begin{array}{l}40 \mathrm{MHz} \\
\text { Bragg } \\
\text { shift }\end{array}$ & $\begin{array}{l}\text { Sign bit } \\
\text { gene ated on } \\
\text { each } \\
\text { measurement }\end{array}$ & $\begin{array}{c}\text { Sign bit } \\
\text { generated on } \\
\text { each } \\
\text { measurement }\end{array}$ \\
\hline
\end{tabular}



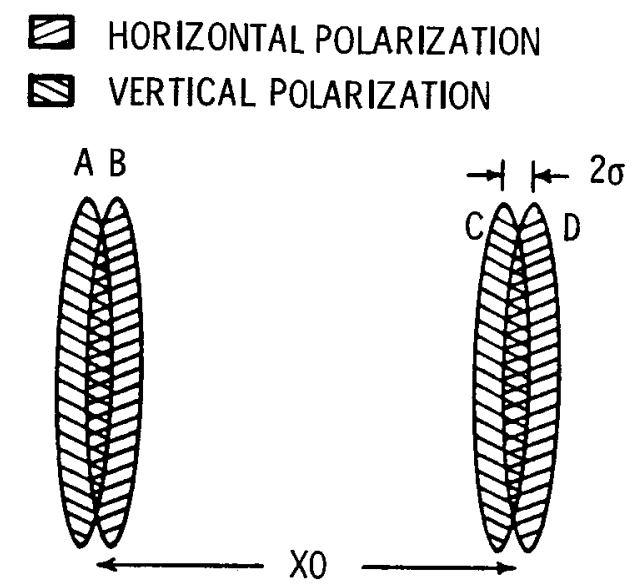

CS $-85-3324$

FIGURE 1. - FOUR SPOT TOFA MEASUREMENT VOLUME GEOMETRY. THE ORTHOGONALLY POLARIZED SPOTS OVERLAP BY $2 \sigma$. THE SPOT PAIRS ARE SEPARATED BY $X O$.

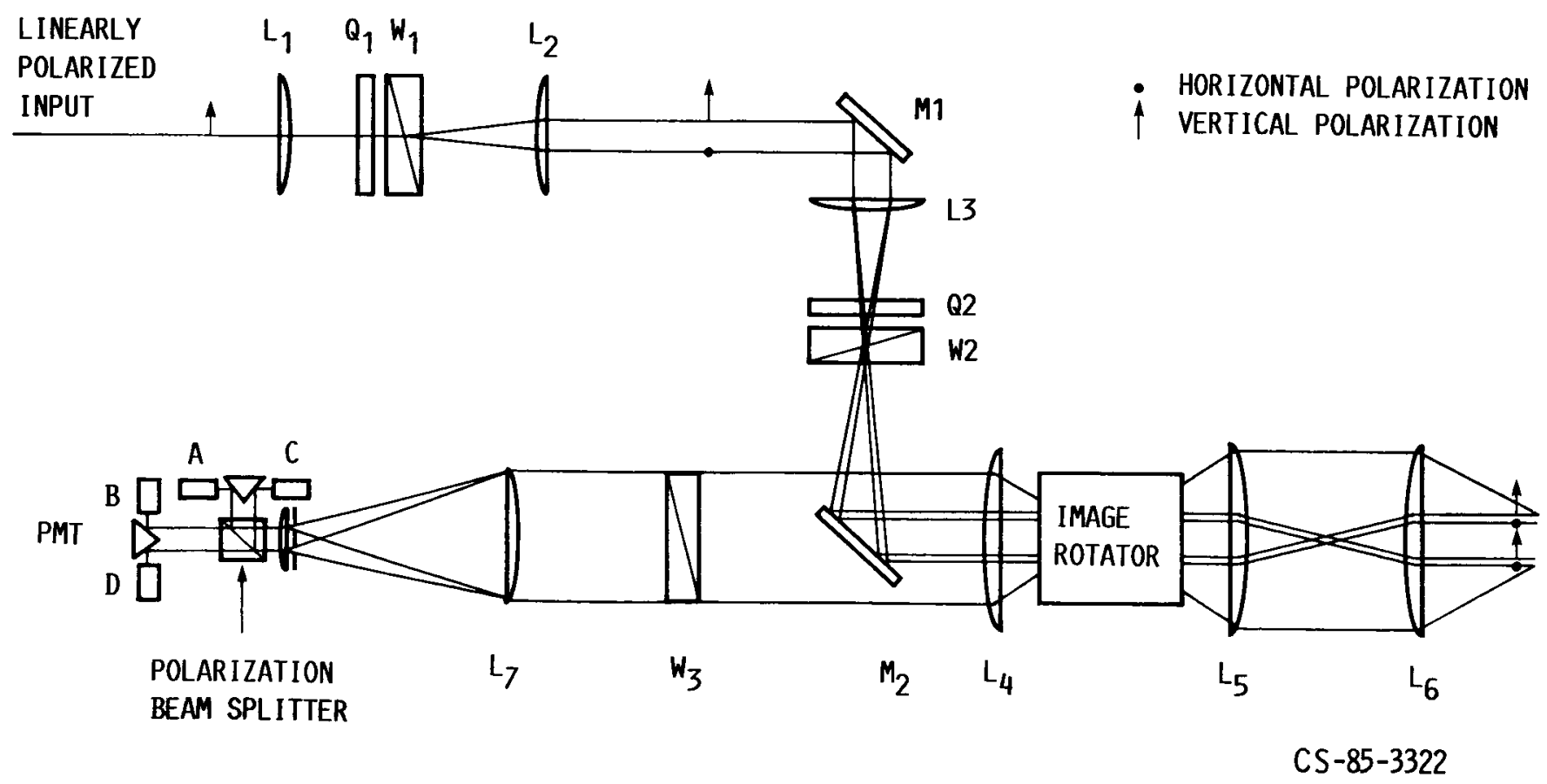

FIGURE 2. - SCHEMATIC VIEW OF THE TRANSMITTING AND RECEIVING OPTICS. 


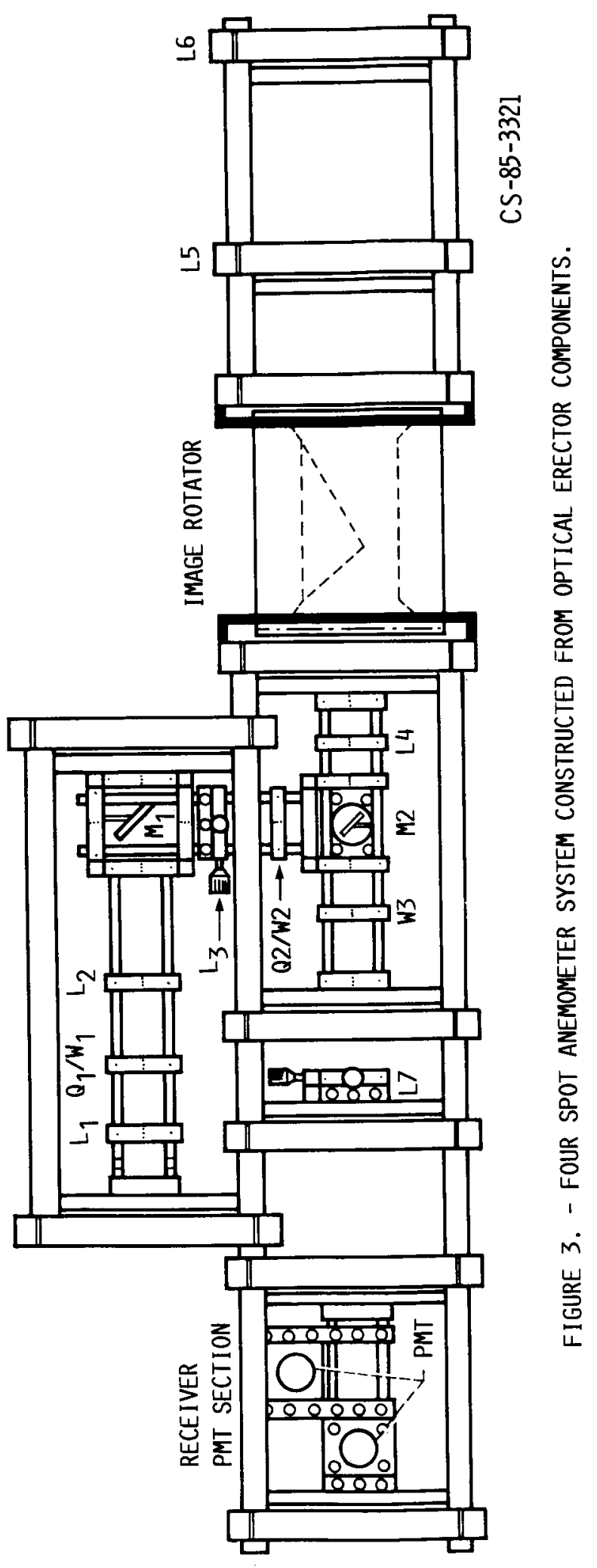


MEASUREMENT STATIONS SHOWN ON BLADE

$$
Y=-17.5 \text { MM (CONSTANT) }
$$

\#1: $Z=-2 M M$

42: $Z=0 \mathrm{MM}$

\#3: $Z=2.0 \mathrm{MM}$

\#4: $Z=4.0 \mathrm{mM}$

\#5: $Z=6.0 \mathrm{MM}$ SURFACE

\#6: $\quad Z=8.0 \mathrm{mM}$

\#7: $Z=10.0 \mathrm{MM}$

\#8: $\quad Z=12.0 \mathrm{MM}$

$\# 9: \quad Z=20.0 \mathrm{mM}\left\{\begin{array}{l}\text { FLOW ANGLE } \\ \text { MEASUREMENTS }\end{array}\right.$

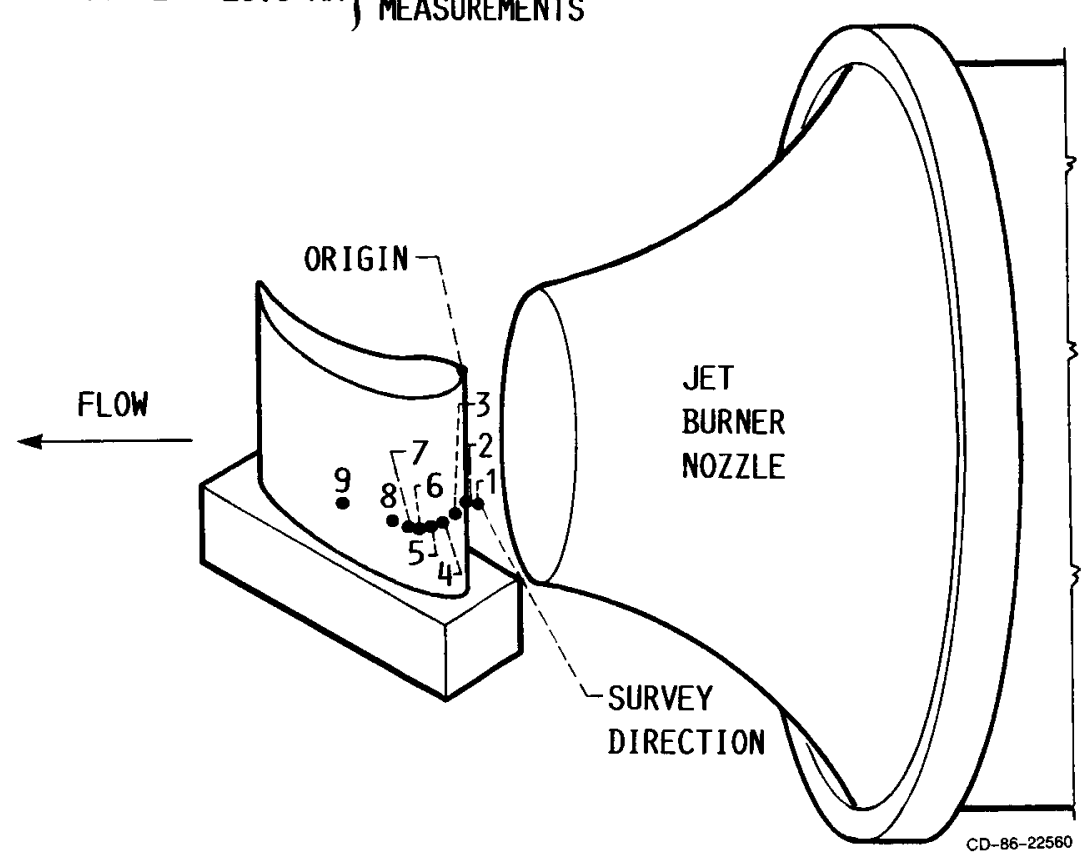

FIGURE 4. - TURBINE BLADE IN HOT CROSS FLOW. THE X-PLANE SURVEY MEASUREMENT STATIONS ARE SHOWN ALONG THE $Z$ DIRECTION. THE CORE TURBINE BLADE WAS MOUNTED TO WITHIN $2^{0}$ OF THE INLET DESIGN ANGLE. 


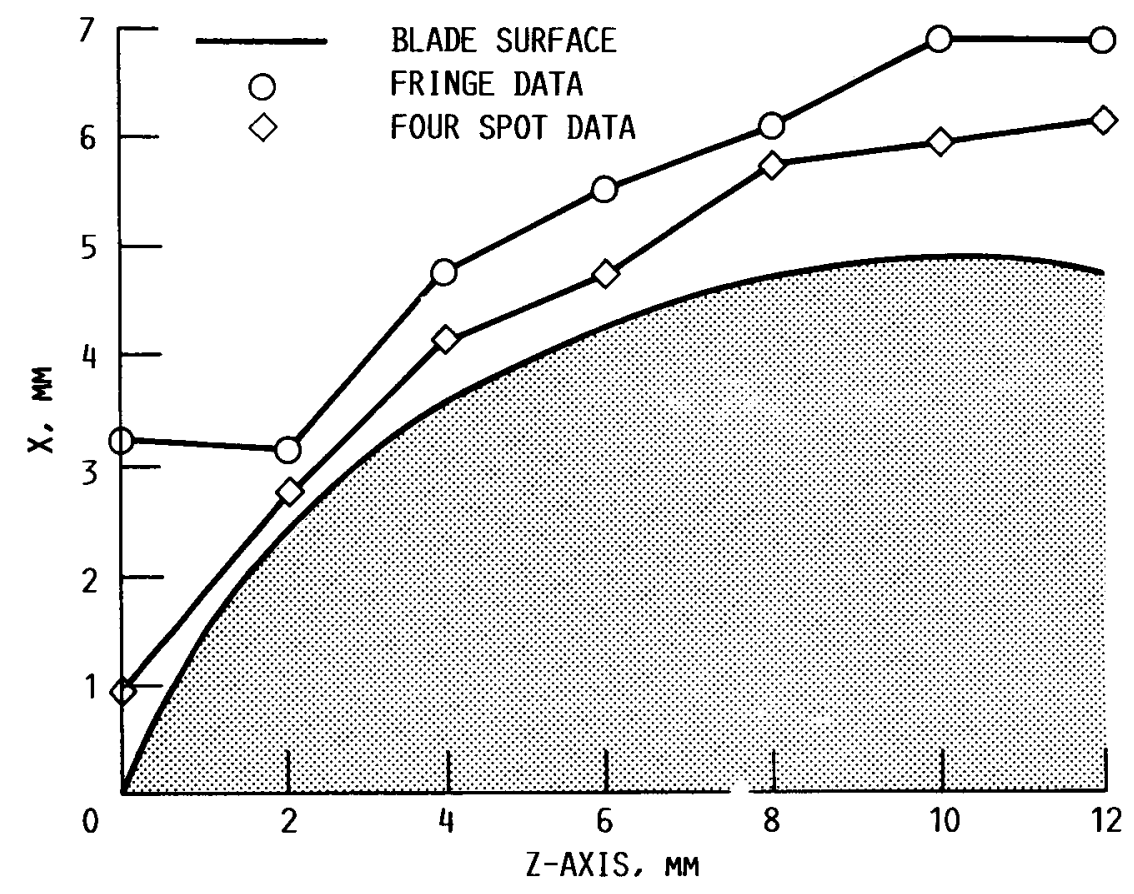

FIGURE 5. - COMPARISON OF SURFACE PROXIMITY MEASUREMENTS FOR THE LFA AND FOUR SPOT TOFA. THE BLADE SURFACE IS SHOWN AS DETERMINED BY THE SURFACE LOCATION SURVEYS. THE FOUR SPOT CONSISTENTLY SHOWED SUPERIOR SURFACE PROXIMITY PERFORMANCE OVER THE LFA. BLADE LEADING EDGE AT ORIGIN. 

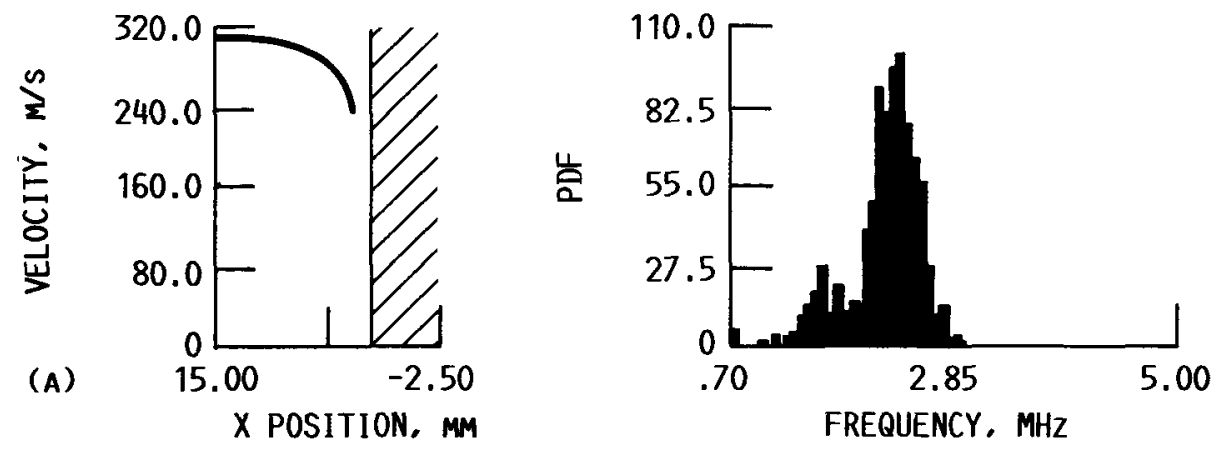

$X$ LOC. $=4.60 \mathrm{MM}$ $Y$ LOC. $=-17.50 \mathrm{MM}$

$Z$ LOC. $=2.00 \mathrm{MM}$

BLADE SURFACE $=3.27 \mathrm{MM}$

$$
\begin{aligned}
& \text { FRQ MEAN }=2.26 \mathrm{MHz} \\
& \text { STD DEV }=16.54 \mathrm{\%} \\
& \text { DATA RATE }=0.27727 \mathrm{kHz} \\
& \text { PMT CURR }=1.0 \mathrm{UA} \\
& \text { LOW PASS }=100.00 \mathrm{MHz} \\
& \text { VELOCITY }=\text { FREQ }(\mathrm{MHz}) \times 106 \mu \mathrm{M}
\end{aligned}
$$
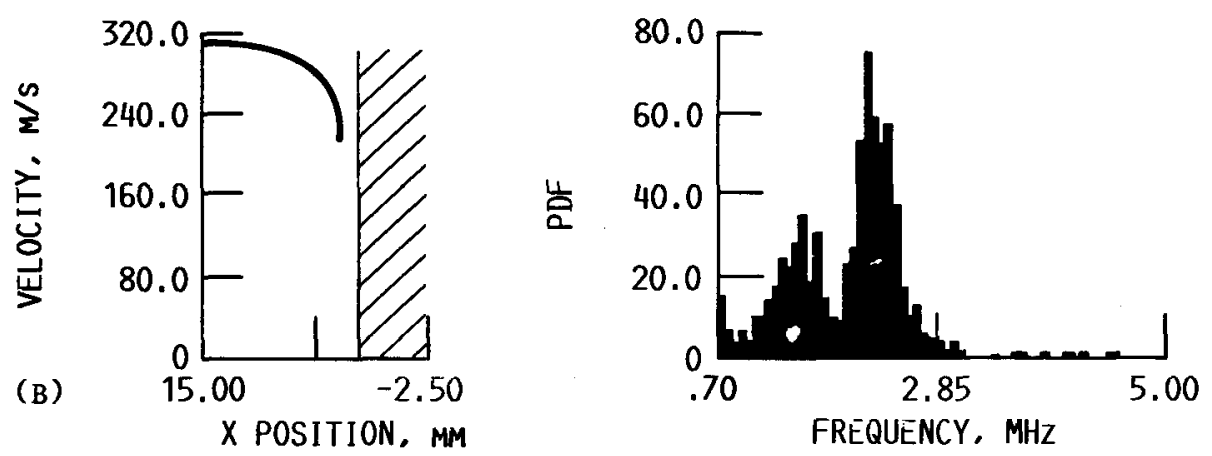

$$
\begin{aligned}
& X \text { LOC. }=4.40 \mathrm{mM} \\
& Y \text { LOC. }=-17.50 \mathrm{mM} \\
& Z \text { LOC. }=2.00 \mathrm{mM} \\
& \text { BLADE SURFACE }=3.27 \mathrm{mM}
\end{aligned}
$$

$$
\begin{aligned}
& \text { FRQ MEAN }=2.01 \mathrm{MHz} \\
& \text { STD DEV }=25.93 \% \\
& \text { DATA RATE }=0.17724 \mathrm{kHz} \\
& \text { PMT CURR }=1.0 \mathrm{UA} \\
& \text { LOW PASS }=100.00 \mathrm{MHz} \\
& \text { VELOCITY }=\text { FREQ }(\mathrm{MHZ}) \times 106 \mu \mathrm{M}
\end{aligned}
$$

FIGURE 6. - THESE FIGURES SHOW THE ACCUMULATED ON-LINE VELOCITY SURVEYS AND THE MEASURED PDFS AT EACH MEASUREMENT POSITION.

THIS DATA WAS TAKEN 2 MM DOWNSTREAM OF THE BLADE LEADING EDGE. THE BLADE SURFACE LOCATION IS INDICATED IN THE FIGURES. THE FLOW OSCILLATIONS BECOME EVIDENT IN 6(A). THE MAGNITUDE OF THE OSCILLATIONS GROWS, REACHING A MAXIMUM IN 6(D), AND THEN FALLING OFF. 

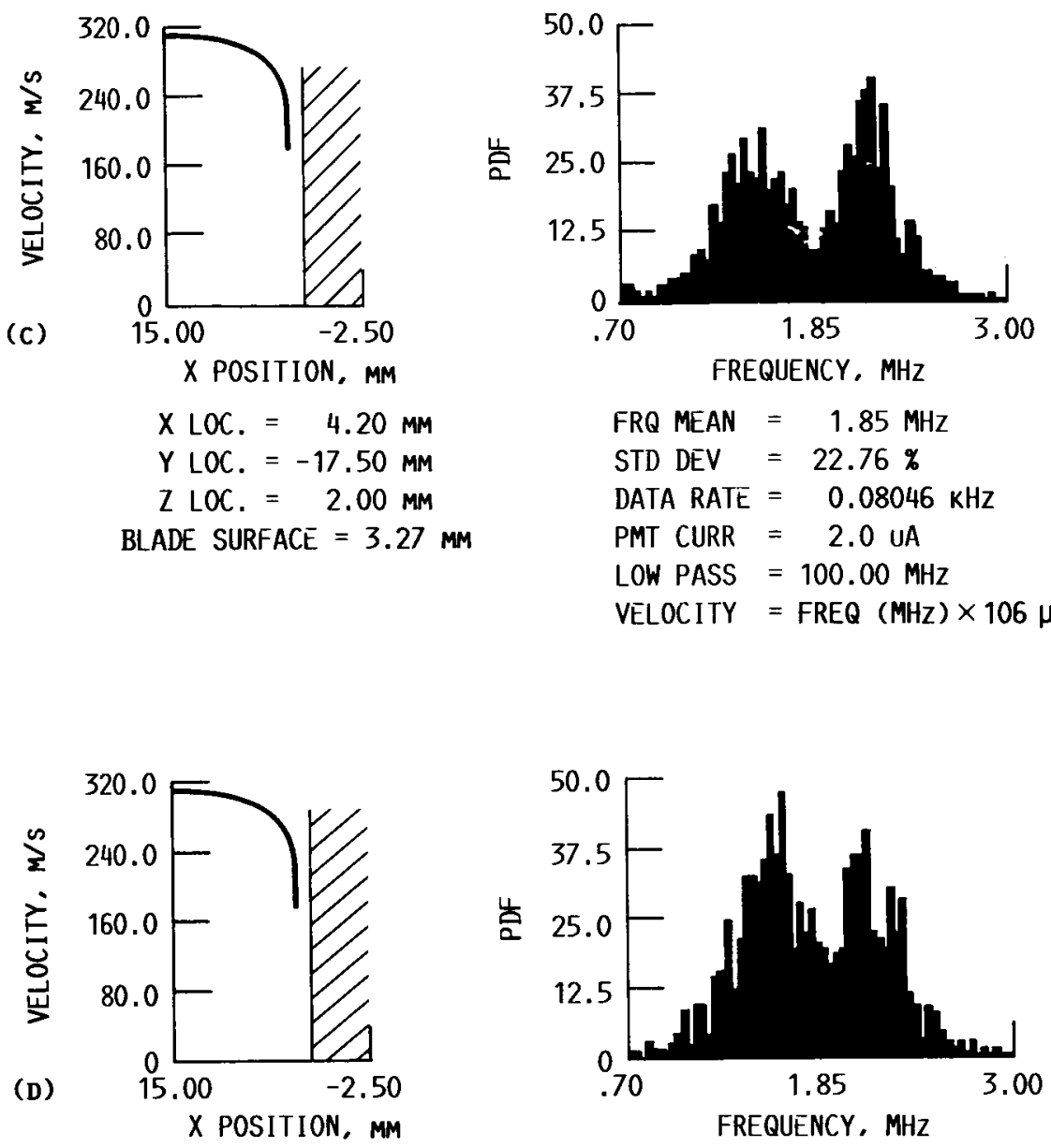

$X \angle O C .=4.00 \mathrm{mM}$

$Y$ LOC. $=-17.50 \mathrm{MM}$

$Z$ LOC. $=2.00 \mathrm{MM}$

BLADE SURFÄCE $=3.27 \mathrm{mM}$

FRQ MEAN $=1.85 \mathrm{MHz}$

STD DEV $=22.76 \%$

DATA RATE $=0.08046 \mathrm{kHz}$

PMT CURR $=2.0 \mathrm{UA}$

LOW PASS $=100.00 \mathrm{MHz}$

VELOCITY $=$ FREQ $(\mathrm{MHZ}) \times 106 \mu \mathrm{M}$

FRQ MEAN $=1.82 \mathrm{MHz}$

STD DEV $=20.87 \%$

DATA RATE $\rightarrow 0.00970 \mathrm{kHz}$

PMT CURR $=2.0 \mathrm{UA}$

LOW FASS $=100.00 \mathrm{MHZ}$

VELOCITY $=$ FREQ $(\mathrm{MHZ}) \times 106 \mu \mathrm{M}$
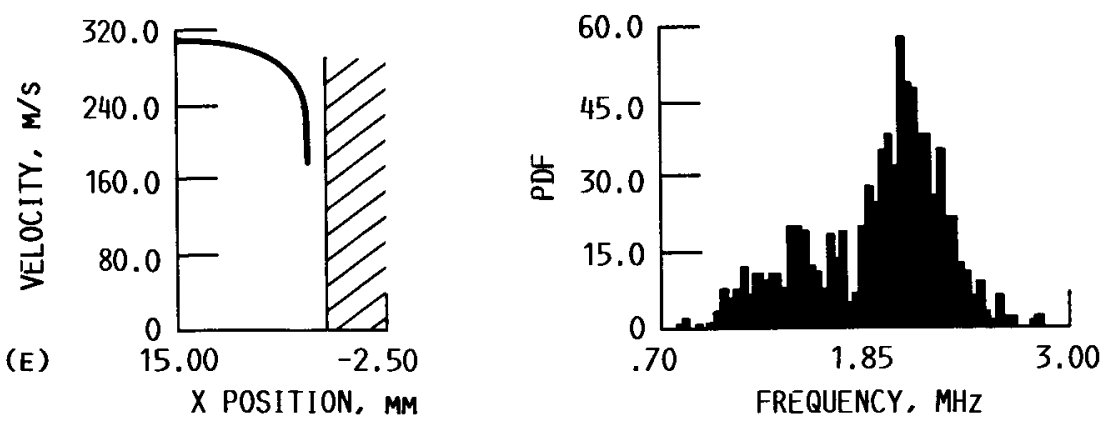

$X$ LOC. $=3.80 \mathrm{MM}$

$Y L O C .=-17.50 \mathrm{mM}$

$Z$ LOC. $=2.00 \mathrm{mM}$

BLADE SURFACE $=3.27 \mathrm{mM}$

FIGURE 6. - CONCLUDED. 


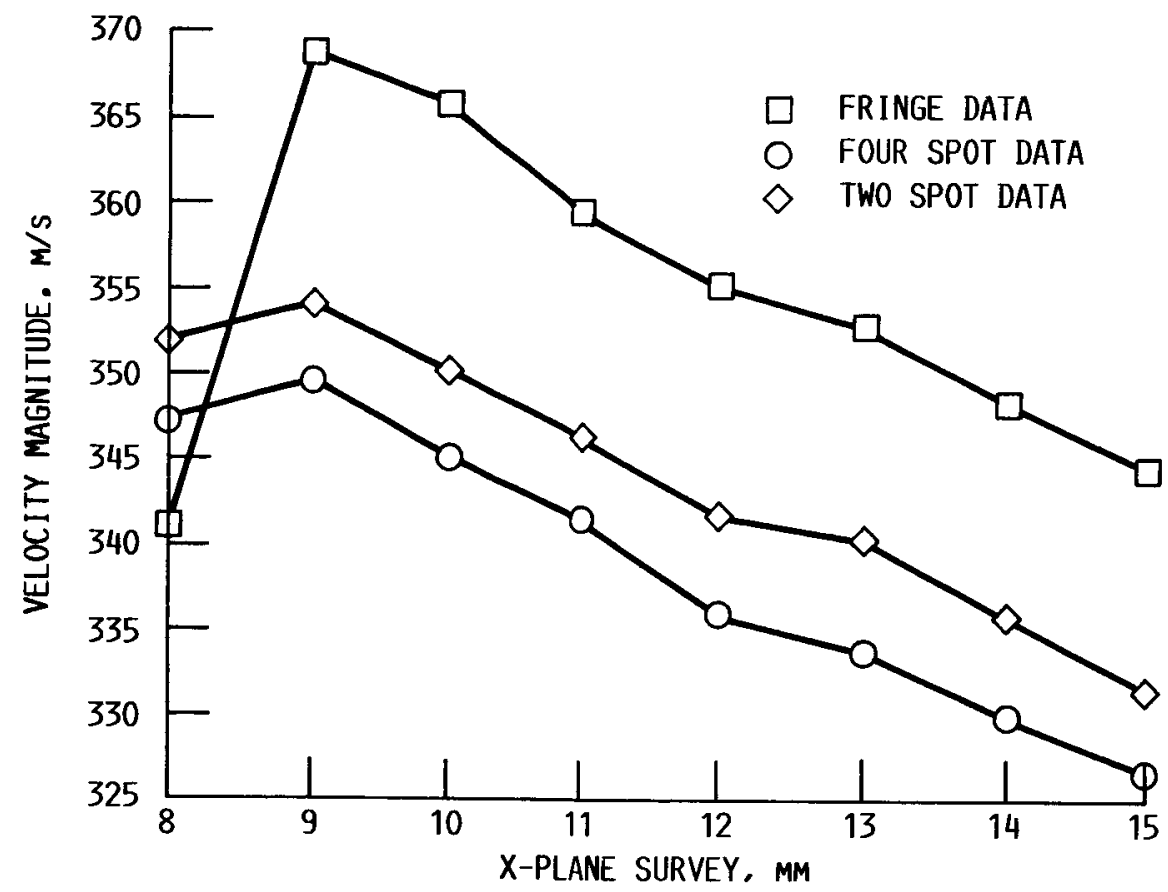

FIGURE 7. - COMPARISON OF THE MEASURED VELOCITY MAGNITUDE FOR THE LFA, FOUR SPOT, AND TWO SPOT SYSTEMS. THE DATA WAS TAKEN 20 MM DOWNSTREAM FROM THE BLADE LEADING EDGE.

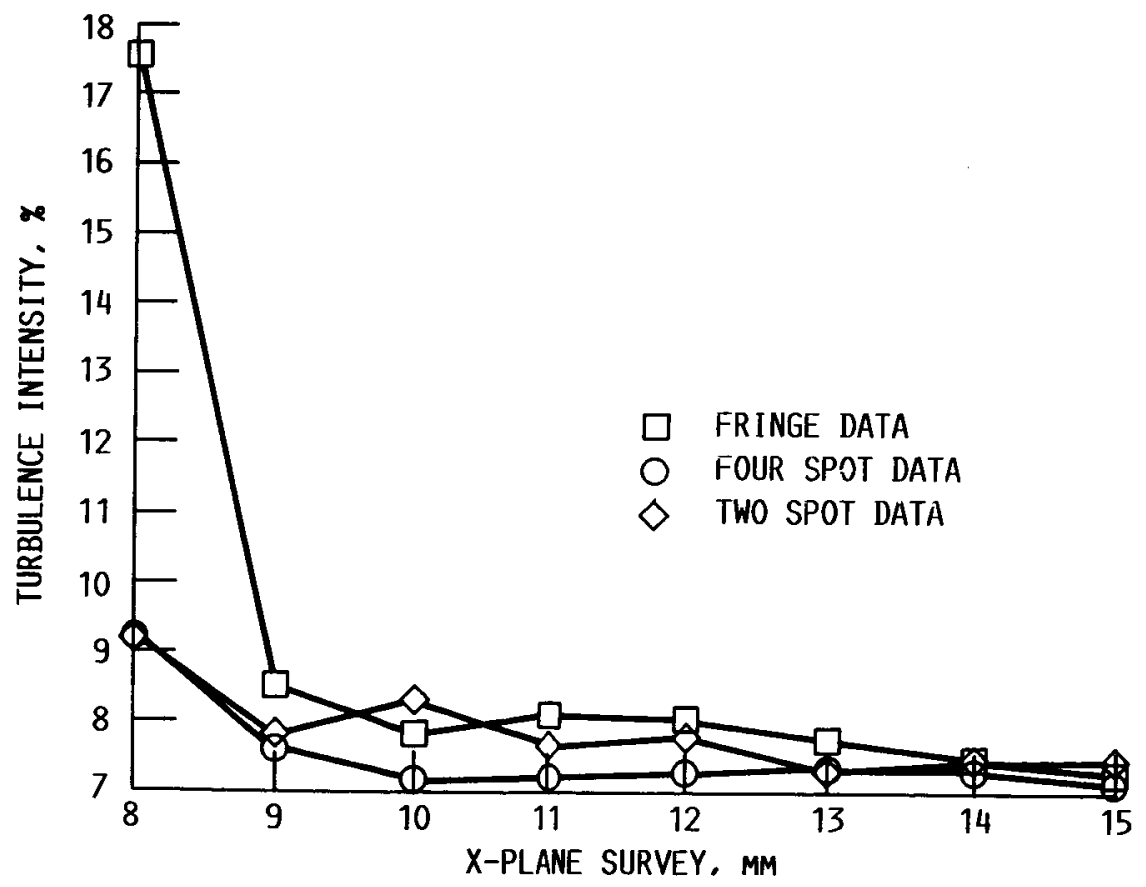

FIGURE 8. - COMPARISON OF THE MEASURED TURBULENCE INTENSITY FOR THE LFA, FOUR SPOT. AND TWO SPOT SYSTEMS. THE TURBULENCE INTENSITY CONTAINED BOTH THE MEASUREMENT UNCERTAINTY AND THE INHERENT INSTRUMENT ERROR. 


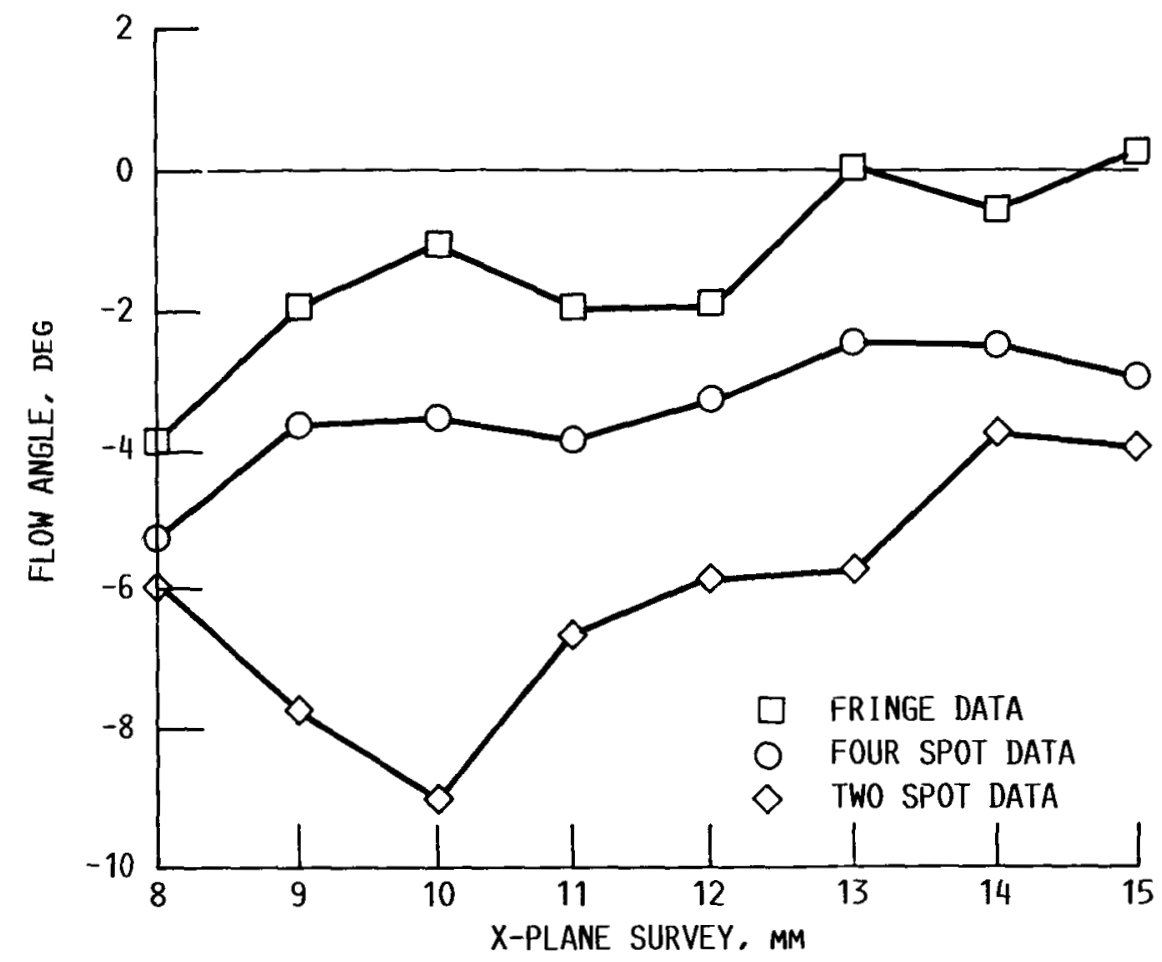

FIGURE 9. - COMPARISON OF THE MEASURED FLOW ANGLES FOR THE LFA, FOUR SPOT, AND TWO SPOT SYSTEMS. THE TWO SPOT FLOW ANGLE ESTIMATES WERE OBTAINED USING THE MEASURED MEAN VELOCITIES AS A FUNCTION OF MEASUREMENT ANGLE. THE SHAPE OF THE LFA AND FOUR SPOT DATA AGREED FAIRLY CLOSELY, EXCEPT FOR AN OFFSET ERROR FROM THE CALIBRATION PROCEDURE. THE TWO SPOT DATA SHOWS A RANDOM FLUCTUATION POSSIBLY DUE TO THE HIGH TURBULENCE FLOW. 


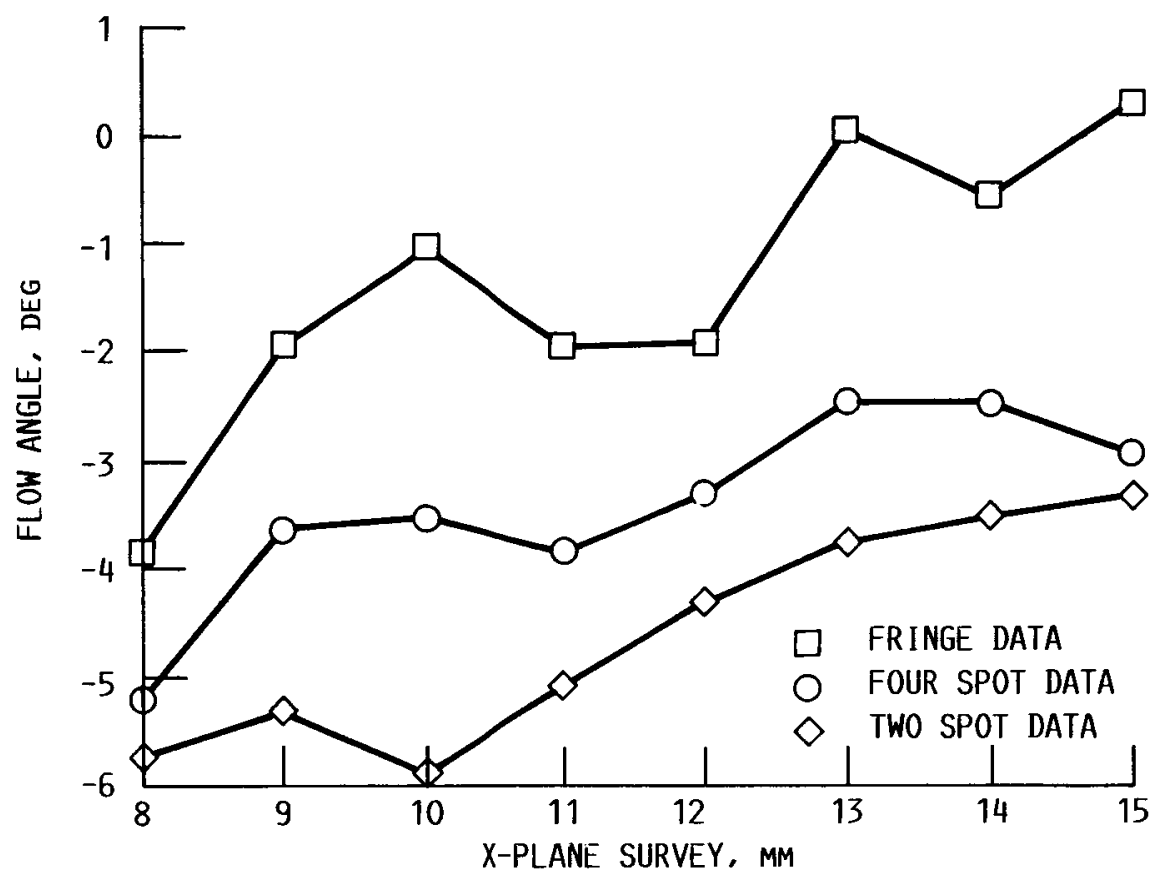

FIGURE 10. - COMPARISON OF THE MEASURED FLOW ANGLES FOR THE LFA, FOUR SPOT. AND TWO SPOT SYSTEMS. THE TWO SPOT FLOW ANGLE ESTIMATES WERE OBTAINED USING THE DATA RATES INSTEAD OF THE MEASURED MEAN VELOCITIES. THE TWO SPOT DATA STILL. SHOWS A DEVIATION FROM THE LFA AND FOUR SPOT FLOW ANGLE DATA. 


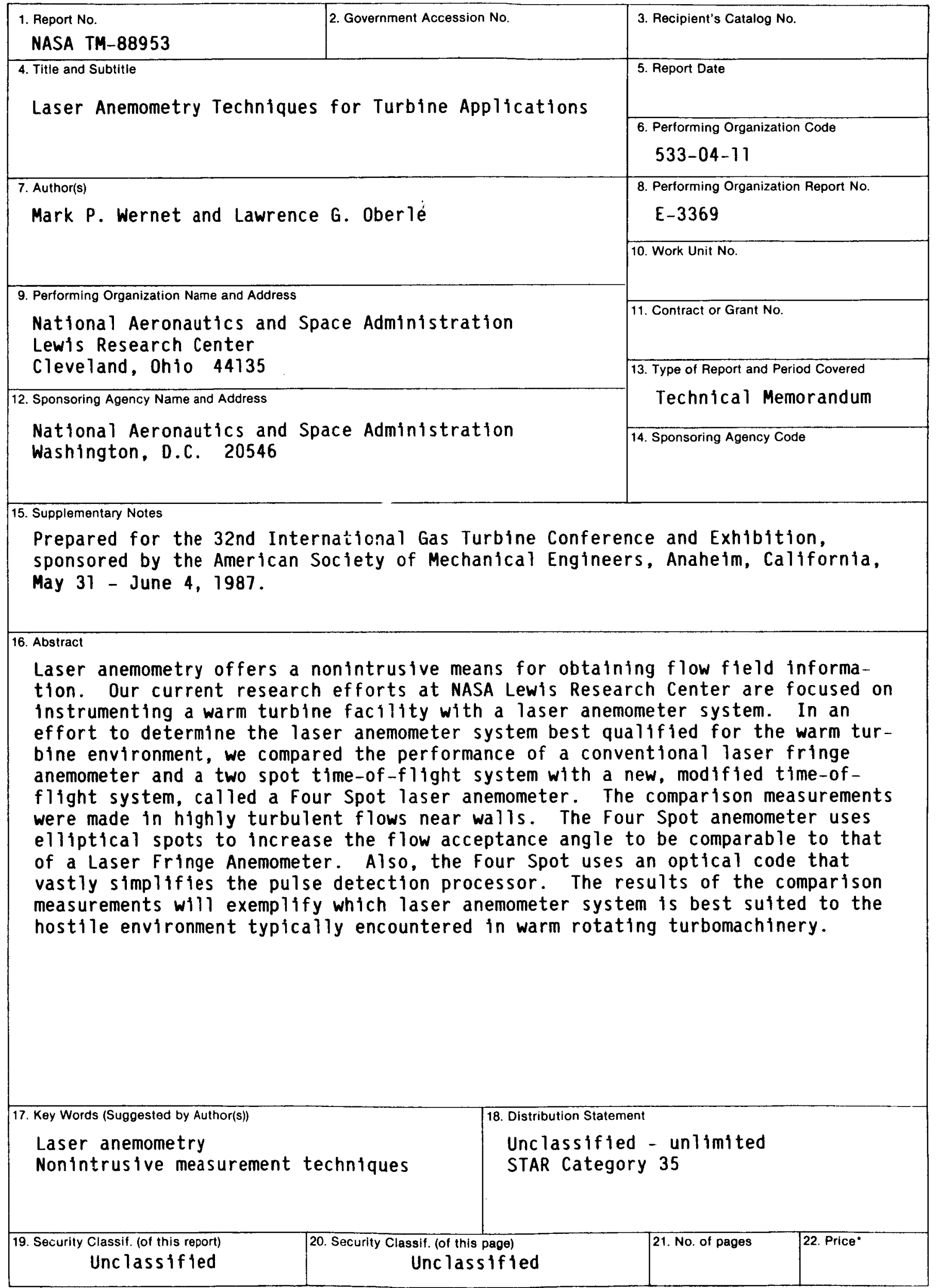

"For sale by the National Technical Information Service, Springfield, Virginia 22161 